\title{
Atmospheric neutrinos in next-generation xenon and argon dark matter experiments
}

\author{
Jayden L. Newstead $\odot,{ }^{1,2,3}$ Rafael F. Lang $\odot,{ }^{2}$ and Louis E. Strigari ${ }^{4}$ \\ ${ }^{1}$ Department of Physics, Arizona State University, Tempe, Arizona 85287, USA \\ ${ }^{2}$ Department of Physics and Astronomy, Purdue University, West Lafayette, Indiana 47907, USA \\ ${ }^{3}$ School of Physics, The University of Melbourne, Victoria 3010, Australia \\ ${ }^{4}$ Mitchell Institute for Fundamental Physics and Astronomy, Department of Physics and Astronomy, \\ Texas A\&M University, College Station, Texas 77845, USA
}

(Received 25 February 2020; accepted 6 December 2021; published 22 December 2021)

\begin{abstract}
We study the sensitivity of future xenon- and argon-based dark matter and neutrino detection experiments to low-energy atmospheric neutrinos. Not accounting for experimental backgrounds, the primary obstacle for identifying nuclear recoils induced by atmospheric neutrinos in xenon is the tail of the electron recoil distribution due to $p p$ solar neutrinos. We use the NEST code to model the solar and atmospheric neutrino signals in a xenon detector and find that an exposure of 700 tonne-years will produce a $5 \sigma$ detection of atmospheric neutrinos. We explore the effect of different detector properties and find that a sufficiently long electron lifetime is essential to the success of such a measurement.
\end{abstract}

DOI: 10.1103/PhysRevD.104.115022

\section{INTRODUCTION}

Multi-ton liquid noble dark matter direct detection experiments will soon be sensitive to coherent neutrinonucleus elastic scattering (CE $\nu \mathrm{NS}$ ) from astrophysical neutrinos, specifically from the Sun, the atmosphere [1,2], and possibly from Galactic [3] and diffuse supernovae [4]. Identifying these neutrinos is an important goal for neutrino physics [5], and is an important milestone for future multi-purpose dark matter detectors [6,7]. A measurement of the low-energy component of atmospheric neutrinos (below $100 \mathrm{MeV}$ ) has not yet been performed, doing so will validate and improve models of their creation (e.g., [8]) which in turn are used to constrain new physics searches [9].

For spin-independent dark matter-nucleus interactions, the nuclear recoil spectrum from a $\sim 6 \mathrm{GeV}$ dark matter particle with cross section $\approx 10^{-45} \mathrm{~cm}^{2}$ mimics the spectrum from the ${ }^{8} \mathrm{~B}$ component of the solar neutrino flux. The detailed sensitivity to solar neutrinos has been the subject of several studies, from the perspective of both a neutrino signal and a background to dark matter detection $[1,2,10$ 13]. For atmospheric neutrinos, a $\sim 100 \mathrm{GeV}$ dark matter particle with cross section $\lesssim 10^{-48} \mathrm{~cm}^{2}$ will mimic the recoil spectrum from atmospheric neutrinos. However, because of the larger exposures required to gain sensitivity to atmospheric neutrinos, understanding this component as either a neutrino signal or a dark matter background has been subject to less scrutiny in the literature. Additionally, neutrino-electron scattering of solar neutrinos can be a significant background in detectors with imperfect electronic/nuclear recoil discrimination, even at rejection rates of $99.5 \%$ [14].

In this paper, we undertake a study of the discovery sensitivity for atmospheric neutrinos in multitonne scale xenon and argon dark matter experiments. We focus on identifying the neutrino-induced nuclear recoil signal in the presence of backgrounds. For xenon detectors the main background arises from electron recoils from the $p p$ component of the solar neutrino flux. Previous studies have estimated the atmospheric neutrino event rate to be order unity for $\sim 20$ tonne-year scale exposures $[1,4]$. Here, we extend upon these results and perform simulations of the nuclear recoil signal in xenon induced by atmospheric neutrinos in a realistic detection configuration using the Noble Element Simulation Technique (NEST) package $[15,16]$.

This paper is organized as follows. In Sec. II we discuss the characteristics of the atmospheric neutrino signal in future xenon and argon experiments. In Sec. III we discuss the NEST simulation for xenon targets and present our statistical method for determining the significance of the neutrino signal as a function of exposure. In Sec. IV we present our primary results, and conclude in Sec. V.

\section{ATMOSPHERIC NEUTRINO SIGNAL}

The atmospheric neutrino flux has been calculated down to energies of $10 \mathrm{MeV}$ using the FLUKA code [17]. At these energies, the flux originates mostly from pion decay, so that the flavor composition is $\sim 2 / 3$ muon flavor and $\sim 1 / 3$ electron flavor. The sub-GeV normalization of the 
atmospheric neutrino flux has not been directly measured, and there are theoretical uncertainties that arise from several physical processes. One such uncertainty arises from the fact that the cosmic ray flux at the top of the Earth's atmosphere differs from the cosmic ray flux in the interstellar medium. A second uncertainty is from the geomagnetic field, which induces a cut-off in the lowenergy cosmic ray spectrum. Detailed modeling of both of these effects implies that for energies $\lesssim 100 \mathrm{MeV}$, the uncertainty on the predicted atmospheric neutrino flux is approximately $20 \%$ [18]. Due in particular to the cutoff in the rigidity of cosmic rays induced by the Earth's geomagnetic field at low energies, the atmospheric neutrino flux is larger for detectors that are nearer to the poles. For example, the low energy $\nu_{\mu}$ flux is about three times larger at Sudbury (latitude $46.5^{\circ}$ ) than it is at Kamioka (latitude $\left.36.2^{\circ}\right)$ [18].

In Fig. 1, we show the total atmospheric neutrino flux, summing over flavor and matter/antimatter, at average solar activity, for the latitude of the Gran Sasso underground laboratory (LNGS). Since LNGS is a possible location for future Generation-3 xenon or argon experiments, and since such experiments are expected to run for more than a decade, we use this flux as shown in Fig. 1 for all following calculations.

Atmospheric neutrinos interact via $\mathrm{CE} \nu \mathrm{NS}$ in a xenon or argon dark matter detector. $\mathrm{CE} \nu \mathrm{NS}$ is a standard model neutral-current process whose differential cross section can be calculated as:

$$
\frac{d \sigma}{d E_{R}}=\frac{G_{F}^{2} m_{T}}{\pi} Q_{w}^{2}\left(1-\frac{m_{T} E_{R}}{2 E_{\nu}^{2}}\right) F^{2}\left(E_{R}\right)
$$



FIG. 1. The solar and atmospheric neutrino flux in the $E_{\nu}=$ 10-100 MeV range. The vertical dashed lines show the corresponding neutrino energies above which xenon and argon detectors are sensitive to, given their nuclear recoil energy thresholds ( $E_{R}=3 \mathrm{keV}$ for xenon, $E_{R}=25 \mathrm{keV}$ for argon). Also shown is the neutrino energy range above which Super-K is sensitive to [8]. where $G_{F}$ is Fermi constant, $m_{T}$ and $Q_{w}$ are the mass and weak charge of the target nuclei, $E_{R}$ is the nuclear recoil energy and $E_{\nu}$ is the incoming neutrino energy. The form factor, $F\left(E_{R}\right)$, accounts for the loss of coherence at larger momentum transfers and is the dominant source of uncertainty in this cross section, contributing around 5\% to the rate normalization for neutrinos in this energy range. Here, we take the form factor to be the one proposed by Helm [19]. The coherent nature of the interaction implies a scaling of the cross section with the number of nucleons squared, but the relatively small weak charge of the proton means that the scaling is closer to the number of neutrons squared. This implies that large atomic mass targets are favored for their neutron-rich nuclei.

The differential event rate per unit detector mass can be calculated from

$$
\frac{d^{2} R}{d E_{\nu} d E_{R}}=\frac{1}{m_{T}} \frac{d \sigma}{d E_{R}} \frac{d \phi_{\nu, i}}{d E_{\nu}} \Theta\left(E_{R, \max }\left(E_{\nu}\right)-E_{R}\right)
$$

where $\phi_{\nu, i}$ is the $i$ th neutrino flux, and $\Theta$ is the Heaviside step function which restricts $E_{R}$ to be less than the maximum value, corresponding to back-to-back scattering:

$$
E_{R, \max }=\frac{2 E_{\nu}^{2}}{m_{T}+2 E_{\nu}^{2}}
$$

The total event rate in an energy bin can then be obtained by integrating Eq. (2) over the relevant $E_{R}$ and $E_{\nu}$. The dependence on neutron number is exhibited by comparing the CELNS rate for xenon and argon targets, as shown in Fig. 2, where we have calculated the total rate above a given threshold. The CE $\nu$ NS rate falls sharply with increasing

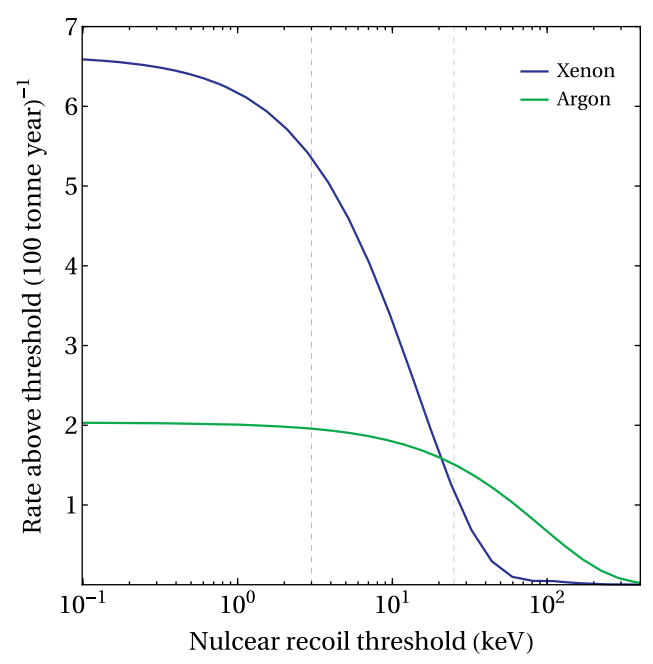

FIG. 2. The integrated event rate of atmospheric neutrino $\mathrm{CE} \nu \mathrm{NS}$ above a given threshold in xenon (blue) and argon (green) detector targets. The vertical dashed lines correspond to illustrative thresholds of $3 \mathrm{keV}$ and $25 \mathrm{keV}$ for xenon and argon, respectively. 


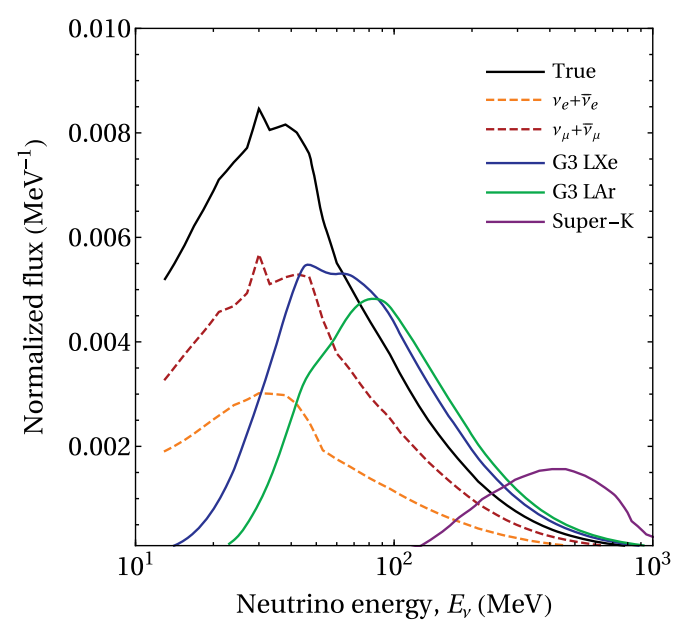

FIG. 3. Normalized energy spectra of atmospheric neutrinos that are detectable by various experiments. The electron and muon-flavored fluxes are indicated with the dashed curves, and the solid black curve is the total atmospheric neutrino flux, summed over all flavors. The features in the neutrino fluxes result from pion and muon decay at rest [21]. Future dark matter experiments will access an atmospheric neutrino energy range that is not accessible to Super-K.

recoil energy due to both loss of coherence and kinematic phase space, highlighting the necessity of a low detector threshold.

From the kinematic limits we can find that a detector sensitive to nuclear recoils in the energy range $\sim 1-50 \mathrm{keV}$ will be sensitive to neutrinos in the energy range $\sim 40-60 \mathrm{MeV}$. More precisely, we can asses the range of energies of atmospheric neutrinos a given detector is sensitive to by integrating Eq. (2) over $E_{R}$ above a specified threshold. The result of this integration as a function of $E_{\nu}$ is given in Fig. 3, indicating the neutrino energy range that a xenon and argon detector with $E_{R} \geq 3 \mathrm{keV}$ and $E_{R} \geq$ $25 \mathrm{keV}$ (respectively) would be sensitive to. For comparison we also show the lowest energy channel (sub-GeV single-ring electron-like events) that Super-Kamiokande was sensitive to in their atmospheric neutrino analysis [8]. As indicated, Super-Kamiokande is sensitive to neutrinos $\gtrsim 100 \mathrm{MeV}$ for their fully contained electronlike events. We note that JUNO would be sensitive to low-energy atmospheric neutrinos through the charged current channel [20].

\section{METHOD}

\section{A. Detector properties}

Dual-phase noble time-projection chambers (TPCs) have proven to be a robust and scalable detector design for direct dark matter searches [22-24]. Xenon (argon) detectors of this design are sensitive to $\mathcal{O}(1 \mathrm{keV})$ nuclear recoils $(\mathcal{O}(10 \mathrm{keV}))$ and provide 3D position reconstruction of events (though this ability is diminished at threshold energies). The position reconstruction allows for detector fiducialization, where one takes advantage in particular of xenon detectors to self-shield, to achieve very low background in the central target volume. This is achieved through the detection of both the scintillation photons and ionization electrons that are produced by interactions in the detector bulk. The prompt scintillation light signal, S1, is measured directly by an array of PMTs on the top and bottom of the detector. The liberated electrons are drifted to the surface of the liquid phase and extracted into the gas phase where, through an avalanche process, they produce the delayed scintillation signal, S2.

We model a future Generation-3 xenon detector as a scaled-up version of the LZ/XENON1T detector, with dimensions scaled to obtain a fiducial region of 100 tonnes. The detector geometry is sketched in Fig. 4 and the dimensions are given in Table I. The detector is modeled using the NESTv2.2 code which simulates the detailed microphysics of the quanta production, recombination and final signal detection for electronic and nuclear recoil events in xenon [16]. We use a series of different detector configurations to investigate the effect of the different detector parameters on the results of the analysis. Two sets of parameter values were used: a baseline value, where the parameter is similar to that already achieved and an enhanced value that we deem achievable based on ongoing work in the community. The purpose of the enhancement is to demonstrate which parameter is limiting the detector performance and therefore would most benefit from future research and development. Most of our baseline parameters were derived from XENON1T which achieved a $g_{1}=$ $0.115 \mathrm{phd} /$ photon (photoelectrons detected per photon), a drift field of $80-120 \mathrm{~V} / \mathrm{cm}$, an electron lifetime of $640 \mu \mathrm{s}$

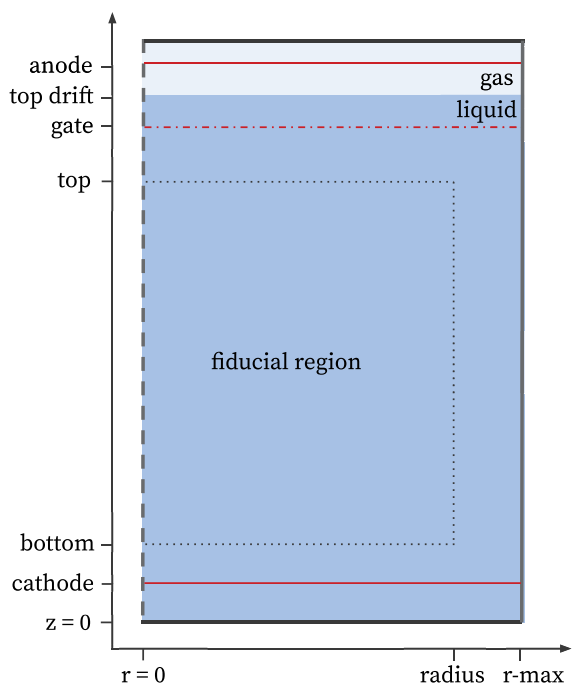

FIG. 4. Cross sectional geometry of a liquid xenon TPC (not to scale), where cylindrical symmetry has been assumed. Dimensions for the 100 tonne fiducial volume detector modeled in this work are given in Table I. 
TABLE I. The 100 tonne xenon detector modeled in this work, the corresponding geometric parameters are shown in Fig. 4. The 100 tonne fiducial volume is based on a liquid xenon density of $\rho=2.8611 \mathrm{~g} / \mathrm{cm}^{3}$.

\begin{tabular}{lc}
\hline \hline Dimension & Position $(\mathrm{m})$ \\
\hline r-max & 1.820 \\
Radius & 1.770 \\
Cathode & 0.200 \\
Bottom & 0.327 \\
Top & 3.878 \\
Gate & 4.000 \\
Top drift & 4.005 \\
Anode & 4.010 \\
\hline \hline
\end{tabular}

$[25,26]$, while $g_{2}$ was taken to approximate the LZ baseline S2 photoelectron yield [27]. All detector parameters are taken to be TPC-average values, we leave a more detailed analysis of position dependent effects to a future analysis. A summary of these parameters and configurations is given in Table II. In all configurations, we take the double-photoelectron detection probability to be $22 \%$ ( 1 phd $=1.22 \mathrm{PE}$ as measured by the PMT), the number of PMTs to be 2400, and we require a 2-fold PMT coincidence for detection. We do not model accidental coincidences from dark counts, which would strongly depend on the dark count rate of the PMT's. This source of noise will affect the detector response at low values of $\mathrm{S} 1$, however it is unlikely our results here as the atmospheric rate is spread over a large energy (and thus S1) range. Further details of the detector parameters, including the PMT properties and geometry, are taken from Refs. [27,28] and held constant for all configurations. All analysis code, configuration files and results are publicly available for download [29].

For comparison we also model a future Generation-3 argon detector. As argon detectors are able to achieve excellent electronic/nuclear recoil discrimination, no detailed detector simulation is required. We instead assume perfect discrimination above nuclear recoil energies of $25 \mathrm{keV}$, i.e., zero electronic recoil background in the region of interest. A threshold of $25 \mathrm{keV}$ represents an optimistic future scenario. For comparison we also show results with a threshold of $55 \mathrm{keV}$ which represents current detector technology. More detailed modeling of an argon detector would be able to account for the roll-off of discrimination ability at low energies, allowing one to further lower the threshold at a cost of efficiency. However, since the atmospheric rate is not strongly dependent on threshold, the small increase in signal (below $25 \mathrm{keV}$ ) would have a correspondingly small effect on the present analysis.

\section{B. Background components}

In this analysis we include all intrinsic backgrounds to an atmospheric neutrino search in xenon, and include the leading extrinsic background ${ }^{222} \mathrm{Rn}$. This seems realistic in light of the current state of the art, with only mild extrapolation needed to a Generation-3 detector which would exhibit superior self-shielding. The irreducible background consist of: electronic recoils from solar $p p$ and ${ }^{7} \mathrm{Be}$ neutrinos, nuclear recoils from solar ${ }^{8} \mathrm{~B}$ and $h e p$ neutrinos, the diffuse supernova neutrino background (dsnb), and the $\nu \nu \beta \beta$ decay of ${ }^{136} \mathrm{Xe}$. The $\nu \nu \beta \beta$ background could be suppressed through depletion of ${ }^{136} \mathrm{Xe}$, as explored in [13]. Here however we assume no depletion, as ${ }^{136} \mathrm{Xe}$ is not the dominant background and will likely be desirable for a $0 \nu \beta \beta$ search [30]. We take the baseline ${ }^{222} \mathrm{Rn}$ background to be $10^{-5}$ dru (events $/ \mathrm{kg} / \mathrm{day} / \mathrm{keV}$ ), just below the expected rate for LZ [31].

In this work, we use calculations of the solar neutrino electronic recoil rate from Ref. [32], which account for a $\sim 23 \%$ suppression of the rate due to atomic binding effects. Additionally, we account for a $\sim 9 \%$ reduction of the charge yield for L-shell electron recoils, as recently observed in electron-capture calibrations of the XELDA detector [33]. This has the effect of widening the solar neutrino electronic recoil band and thus slightly increases the number of electronic recoil background events in the nuclear recoil signal band.

\section{Likelihood analysis}

To evaluate the future potential for discovery and measurement of the atmospheric neutrino flux, we perform

TABLE II. List of xenon detector configurations and their corresponding parameters modelled in NEST. Note that $g_{2}$ is a derived parameter calculated from more fundamental detector parameters, see [29] for the full detector files used for this analysis.

\begin{tabular}{lccccc}
\hline \hline Configuration & $g_{1}(\mathrm{phd} / \gamma)$ & $g_{2}(\mathrm{phd} / \mathrm{e})$ & Drift field $(\mathrm{V} / \mathrm{cm})$ & Electron lifetime $(\mu s)$ & ${ }^{222}$ Rn background (dru) \\
\hline Baseline & 0.12 & 44 & 310 & 650 & $10^{-5}$ \\
Enhanced $g_{1}$ & $\mathbf{0 . 3}$ & 44 & 310 & 650 & $10^{-5}$ \\
Enhanced $g_{2}$ & 0.12 & $\mathbf{1 0 0}$ & 310 & 650 & $10^{-5}$ \\
Enhanced V & 0.12 & 44 & $\mathbf{1 0 0 0}$ & 650 & $10^{-5}$ \\
Enhanced $e$-lifetime & 0.12 & 44 & 310 & $\mathbf{2 5 0 0}$ & $10^{-5}$ \\
Reduced ${ }^{222} \mathrm{Rn}$ & 0.12 & 44 & 310 & 650 & $\mathbf{1 0}^{-\mathbf{6}}$ \\
All enhanced & 0.3 & 100 & 1000 & 2500 & $10^{-6}$ \\
\hline \hline
\end{tabular}



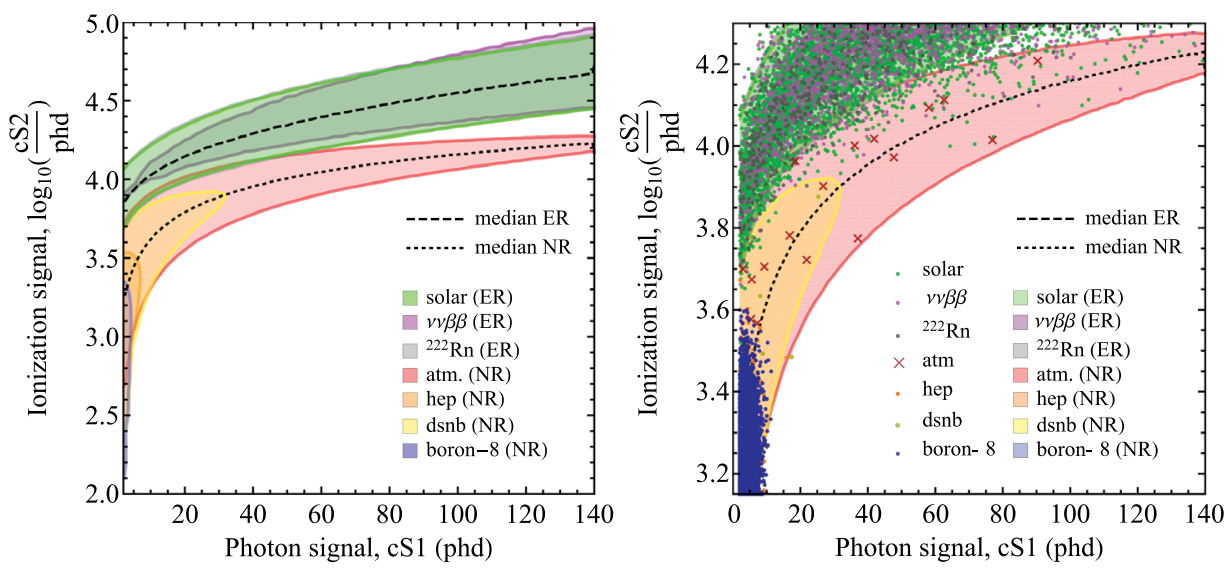

FIG. 5. Results of our NEST model for the "all enhanced" xenon detector configuration. Left: regions which contain $90 \%$ of the events due to the specified source. The dashed (dotted) line shows the median of the nuclear (electronic) recoil band. Right: same as right panel, except now assuming an exposure of 0.2 kilotonne-years, and zooming in on the vertical axis. Points represent simulated events from the indicated flux components.

a binned likelihood analysis on representative (Asimov) data sets [34], simulated with various detector exposures. For the xenon detector we obtained simulated distributions of events in the $\{\mathrm{cS} 1, \mathrm{cS} 2\}$ space using NEST, where $c$ in cS1 refers to the S1 signal after correcting for positiondependent effects. The correction is applied by NEST and here only affects the $\mathrm{S} 2$ signal (via the electron lifetime). To generate these data sets, we perform a Monte Carlo simulation for each detector configuration, with $10^{7}$ events for each source of neutrinos or background component. For the argon detector we perform a single bin analysis, integrating the rate above a chosen threshold.

The distributions obtained for the xenon "all enhanced" detector configuration are shown in the left panel of Fig. 5 at the $90 \%$ confidence level. The separation of signal and background regions can be deceiving since the expected rate for the solar components are orders of magnitude greater than for the atmospheric rate. To visualize the leakage of background events into the expected atmospheric background region we show a sample exposure of 0.2 kilotonne-years (kty) in the right panel of Fig. 5. This sample exposure highlights the futility of trying to define a background free region for an atmospheric neutrino search and why we must rely on statistical discrimination in the $\{\mathrm{cS} 1, \mathrm{cS} 2\}$ plane.

The xenon analysis is performed in a region-of-interest defined by: $2 \leq \mathrm{cS} 1 \leq 120(2 \leq \mathrm{cS} 1 \leq 240$ for the cases with enhanced $\left.g_{1}\right)$ and $2 \leq \log _{10}(\mathrm{cS} 2) \leq 5.7$. Extending this range does not improve our results statistically, so this range is chosen to reduce the computational burden of the analysis by allowing us to focus our simulation on the regions where the signal events are expected. These regions are divided into $N_{\mathrm{S} 1}=120$ and $N_{\mathrm{S} 2}=120$ bins. The Poisson likelihood function is thus,

$$
\mathcal{L}\left(\boldsymbol{n} \mid \lambda\left(\mu^{\prime}\right)\right)=\sum_{i=1}^{N_{S 1}} \sum_{j=1}^{N_{S 2}} \operatorname{Poisson}\left(n_{i, j} \mid \lambda_{i, j}\left(\mu^{\prime}\right)\right)
$$

where Poisson $\left(n_{i, j} \mid \lambda_{i, j}\left(\mu^{\prime}\right)\right)$ is the Poisson probability of observing $n_{i, j}$ events in the $i, j$ bin, given an expected (mean) number of events,

$$
\lambda_{i, j}\left(\mu^{\prime}\right)=b_{i, j}+\mu^{\prime} s_{i, j}
$$

for a given signal strength, $\mu^{\prime}$, expected background, $b$, and signal, $s$.

To calculate the expected statistical significance of discovery for a given exposure and assumed signal strength we use the test statistic

$$
q_{0, A}\left(\mu^{\prime}\right)= \begin{cases}-2 \ln \frac{\mathcal{L}\left(\mu=0 \mid \lambda\left(\mu^{\prime}\right)\right)}{\mathcal{L}\left(\hat{\mu}=1 \mid \lambda\left(\mu^{\prime}\right)\right)} & \hat{\mu} \geq 0 \\ 0 & \hat{\mu}<0\end{cases}
$$

where the expected significance is given by $\sqrt{q_{0, A}}$. We additionally calculate the $90 \%$ confidence interval on the signal strength parameter using the test statistic,

$$
t_{\mu}\left(\mu^{\prime}\right)=-2 \ln \frac{\mathcal{L}\left(\mu^{\prime} \mid \lambda\left(\mu^{\prime}\right)\right)}{\mathcal{L}\left(\hat{\mu} \mid \lambda\left(\mu^{\prime}\right)\right)} .
$$

\section{RESULTS}

To explore the effect of using different xenon detector parameters (as given in Table II) we perform our analysis six times with different configurations. First using the baseline values and then the enhanced values one at a time, and then all together. The expected significance of the 
TABLE III. The expected significance $(\mathrm{Z})$ of the atmospheric neutrino signal for each of the xenon detector configurations with a 1 kty exposure.

\begin{tabular}{lc}
\hline \hline Enhanced parameter & $\mathrm{Z}(\mathrm{cS} 1 \& \mathrm{cS} 2)$ \\
\hline $\mathrm{N} / \mathrm{A}$ & 0.6 \\
$g_{1}$ & 0.7 \\
$g_{2}$ Drift field & 0.6 \\
Electron lifetime & 0.9 \\
${ }^{22} \mathrm{Rn}$ & 5.8 \\
All & 0.9 \\
\hline \hline
\end{tabular}

atmospheric neutrino signal after 1 kty is given for each of the configurations in Table III. While there may be significant correlations between pairs of the parameters, these results clearly show that the parameter with the single largest effect is the mean electron lifetime. This is because most of the parameters are independent of, or scale with, the detector size. This is not the case for the electron lifetime which, if left at $650 \mu \mathrm{s}$, results in far fewer electrons making it to the liquid surface. With a drift velocity of $\sim 2 \mathrm{~mm} / \mu \mathrm{s}$, the enhanced electron lifetime corresponds to an average drift distance of 5 metersenabling most charges to be extracted. Electron lifetimes longer than 5 milliseconds where tested and found to not further improve the detector performance.

The detector configuration with all parameters enhanced experienced an improvement beyond the sum of the individual improvements, indicating that the enhancements are acting synergistically. This can be explained by the observation that increasing $g_{2}$ alone has no improvement on the sensitivity. One would expect that an increase in the gain of the S2 signal would improve electronic/nuclear recoil discrimination by reducing statistical fluctuations in the S2 signal (and also improve position reconstruction). However, if the electron lifetime is small, then there is no signal for $g_{2}$ to amplify, and no improvement is observed. The drift field primarily affects the electron drift velocity and the charge/light yield. The electron drift velocity is increased for higher drift fields, enabling electrons to be drifted from greater depths in the detector within a given electron lifetime. The effect on the charge and light yields are inversely correlated due to conservation of quanta. Larger drift fields extract more electrons from the primary interaction site before they can recombine to produce primary scintillation. This has the greatest effect on events near threshold and so is less relevant to our analysis. The effect of the drift field on these processes is largest at low fields and saturates above $\sim 100 \mathrm{~V}$ [35]. Therefore we do not see a large improvement when going from our baseline of $310 \mathrm{~V} / \mathrm{cm}$ to $1000 \mathrm{~V} / \mathrm{cm}$.

To show the effect of the systematic uncertainty on the projected significance, we perform our significance calculation varying the flux normalization by $\pm 20 \%$, i.e., using Eq. (6) with values of $\mu^{\prime}=0.8,1,1.2$. The results of these calculations for both xenon and argon detectors are shown in Fig. 6. At an exposure of 1 kty, the systematic uncertainty is approximately $\pm 1 \sigma$, which is of the same magnitude as the statistical uncertainty. Figure 7 shows the corresponding confidence interval obtained for a measurement of the atmospheric flux normalization with xenon.

\section{DISCUSSION AND CONCLUSION}

The standard paradigm of dark matter direct detection experiments is to operate as close to the no-background regime as possible. It has long been known that this paradigm would come to an end when the irreducible nuclear recoil backgrounds from neutrinos dominate and
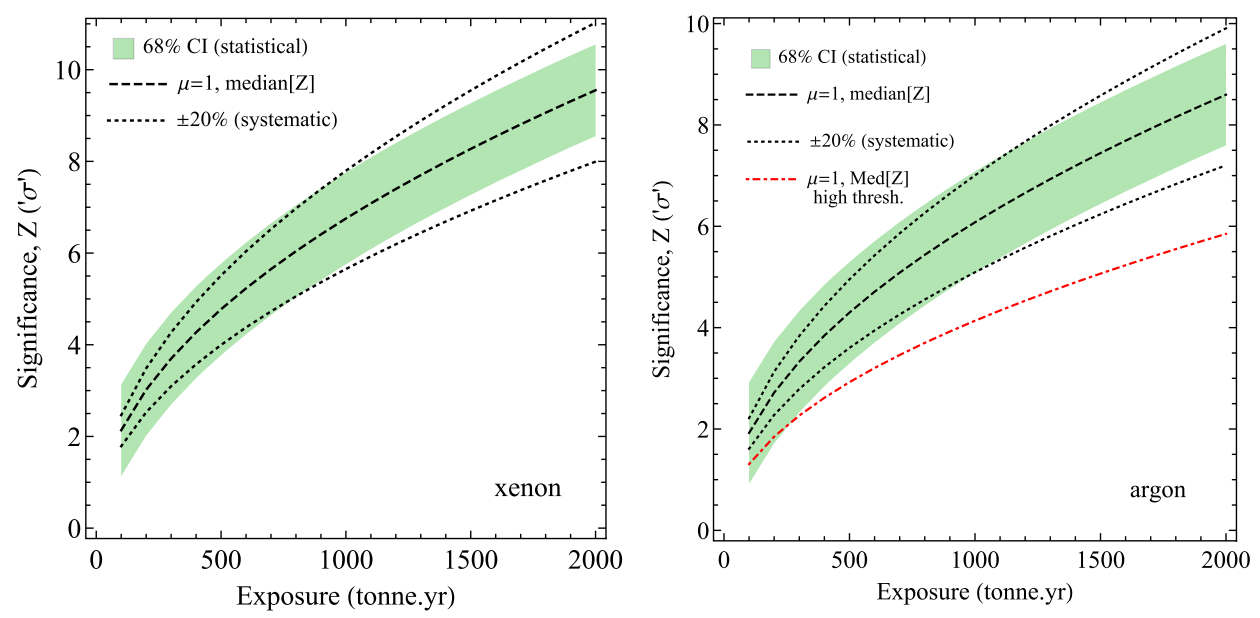

FIG. 6. The median significance (dashed) and 1- $\sigma$ confidence interval, CI, band (green) obtained for xenon (left) and argon (right) detectors as a function of the exposure. The effect of variations in the flux normalization by $\pm 20 \%$ on the median significance is also shown (dotted). For xenon the all enhanced detector configuration was used. For argon a threshold of $25 \mathrm{keV}$ was used, with the effect of raising the threshold to $55 \mathrm{keV}$ shown in red. 


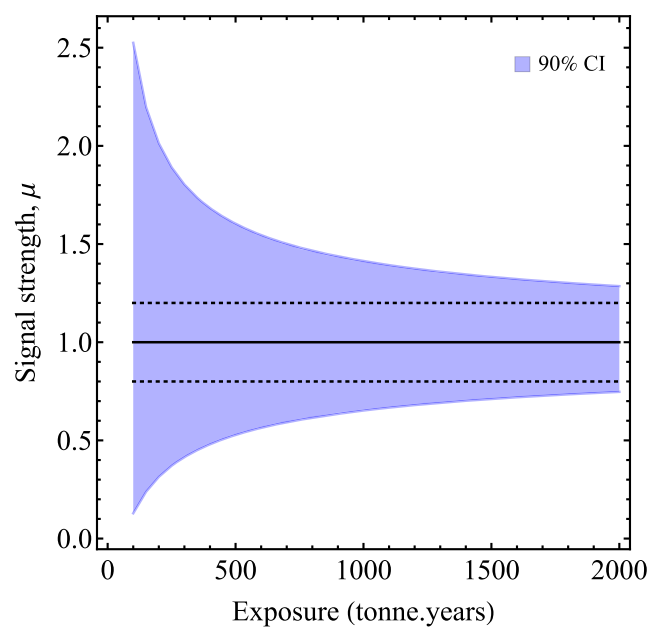

FIG. 7. The $90 \%$ confidence interval of the measured atmospheric flux normalization for xenon (blue). The $20 \%$ systematic uncertainty in the atmospheric flux is shown for comparison (dotted).

sensitivities reach the so-called "neutrino floor." For such nuclear recoil searches, the ability to discriminate and reject the electron recoil background is crucial. In this paper we find that even before reaching the atmospheric background, realistic xenon detectors will observe many solar neutrinos events leaking into the nuclear recoil band (as can be clearly seen in Fig. 5). We have shown that the standard practice of using a 2D likelihood in S1 and S2 provides enough discrimination power to make the first measurement of atmospheric neutrinos. Critically, we have identified that a good electron lifetime is necessary for such a measurement.

We have discussed the prospects for extracting the atmospheric neutrino flux at multitonne scale xenon experiments. Using the best estimates for the low-energy atmospheric neutrino flux, and assuming pure Standard Model physics, we find that an exposure of at least $0.5 \mathrm{kty}$ $(0.7 \mathrm{kty})$ is required to obtain a $5 \sigma$ detection in xenon (argon). This is well within reach of a future Generation-3 detector technology.

The detectors discussed will not only be important for atmospheric neutrinos, but they will provide a new window into solar and supernova neutrinos as well. In addition to focusing on detector specifications, we highlight the need to better understand the systematic uncertainty of the low-energy atmospheric neutrino flux. While a Generation3 xenon detector could be used to make the first measurement of this flux, with the current technology and exposure of less than $2 \mathrm{kty}$, such a measurement would not be able to measure the flux to better than $20 \%$ precision. In this paper we have only studied Standard Model interactions, the presence of non-standard neutrino interactions may alter the flux and scattering rate relative to the predictions presented in this paper [36-38]. Generation-3 experiments, like the ones considered here, will provide complementary information to neutrino experiments which could potentially help identify nonstandard neutrino interactions [9].

\section{ACKNOWLEDGMENTS}

We thank Amanda Depoian for useful comments on this manuscript. The work of L.E. S. is supported by DOE Grant No. de-sc0010813 and that of R. F. L. and J. L. N. by NSF Grant No. PHY-1719271. J. L. N. is supported in part by the Australian Research Council. J. L. N. thanks The Mitchell Institute for their hospitality while a portion of this work was completed.
[1] J. Billard, L. Strigari, and E. Figueroa-Feliciano, Phys. Rev. D 89, 023524 (2014).

[2] J. Billard, L. E. Strigari, and E. Figueroa-Feliciano, Phys. Rev. D 91, 095023 (2015).

[3] R. F. Lang, C. McCabe, S. Reichard, M. Selvi, and I. Tamborra, Phys. Rev. D 94, 103009 (2016).

[4] L. E. Strigari, New J. Phys. 11, 105011 (2009).

[5] B. Dutta and L. E. Strigari, Annu. Rev. Nucl. Part. Sci. 69, 137 (2019).

[6] J. Aalbers et al. (DARWIN Collaboration), J. Cosmol. Astropart. Phys. 11 (2016) 017.

[7] M. Schumann, L. Baudis, L. Btikofer, A. Kish, and M. Selvi, J. Cosmol. Astropart. Phys. 10 (2015) 016.

[8] E. Richard et al. (Super-Kamiokande Collaboration), Phys. Rev. D 94, 052001 (2016).

[9] B. Dutta, R. F. Lang, S. Liao, S. Sinha, L. Strigari, and A. Thompson, J. High Energy Phys. 09 (2020) 106.
[10] J. B. Dent, B. Dutta, J. L. Newstead, and L. E. Strigari, Phys. Rev. D 95, 051701 (2017).

[11] J. B. Dent, B. Dutta, J. L. Newstead, and L. E. Strigari, Phys. Rev. D 93, 075018 (2016).

[12] C. A. J. O'Hare, Phys. Rev. D 102, 063024 (2020).

[13] J. L. Newstead, L. E. Strigari, and R. F. Lang, Phys. Rev. D 99, 043006 (2019).

[14] L. Baudis, A. Ferella, A. Kish, A. Manalaysay, T. Marrodan Undagoitia, and M. Schumann, J. Cosmol. Astropart. Phys. 01 (2014) 044.

[15] M. Szydagis, N. Barry, K. Kazkaz, J. Mock, D. Stolp, M. Sweany, M. Tripathi, S. Uvarov, N. Walsh, and M. Woods, J. Instrum. 6, P10002 (2011).

[16] M. Szydagis, J. Balajthy, J. Brodsky, J. Cutter, J. Huang, E. Kozlova, B. Lenardo, A. Manalaysay, D. McKinsey, M. Mooney, J. Mueller, K. Ni, G. Rischbieter, M. Tripathi, C. Tunnell, V. Velan, and Z. Zhao, NESTCollaboration/nest: 
New, flexible LXe NR yields and resolution model + G4 improvements + linear Noise + much more (2019), 10.5281/zenodo.3357973

[17] G. Battistoni, A. Ferrari, T. Montaruli, and P. R. Sala, Astropart. Phys. 19, 269 (2003); 19, 291(E) (2003).

[18] M. Honda, T. Kajita, K. Kasahara, and S. Midorikawa, Phys. Rev. D 83, 123001 (2011).

[19] R. H. Helm, Phys. Rev. 104, 1466 (1956).

[20] F. An et al. (JUNO Collaboration), J. Phys. G 43, 030401 (2016).

[21] O. L. G. Peres and A. Yu. Smirnov, Phys. Rev. D 79, 113002 (2009).

[22] E. Aprile et al. (XENON Collaboration), Phys. Rev. Lett. 121, 111302 (2018).

[23] X. Cui et al. (PandaX-II Collaboration), Phys. Rev. Lett. 119, 181302 (2017).

[24] D. S. Akerib et al. (LUX Collaboration), Phys. Rev. Lett. 118, 021303 (2017).

[25] E. Aprile et al. (XENON Collaboartion), Phys. Rev. D 100, 052014 (2019).

[26] E. Aprile et al. (XENON Collaboration), Phys. Rev. D 99, 112009 (2019).

[27] B. J. Mount et al., arXiv:1703.09144.

[28] E. Aprile et al. (XENON Collaboration), Eur. Phys. J. C 77, 881 (2017).
[29] J. L. Newstead, Atmospheric neutrinos in a next-generation xenon dark matter experiment (2020), 10.5281/zenodo.3653516.

[30] F. Agostini et al. (DARWIN Collaboration), Eur. Phys. J. C 80, 808 (2020).

[31] D. S. Akerib et al. (LZ Collaboration), Phys. Rev. D 104, 092009 (2021).

[32] J.-W. Chen, H.-C. Chi, C. P. Liu, and C.-P. Wu, Phys. Lett. B 774, 656 (2017).

[33] D. Temples, E. C. Dahl, H. W. Lippincott, B. Baxter, A. Monte, J. McLaughlin, and J. Phelan, Phys. Rev. D 104, 112001 (2014).

[34] G. Cowan, K. Cranmer, E. Gross, and O. Vitells, Eur. Phys. J. C 71, 1554 (2011); 73, 2501(E) (2013).

[35] E. Hogenbirk, M. Decowski, K. McEwan, and A. Colijn, J. Instrum. 13, P10031 (2018).

[36] D. Aristizabal Sierra, N. Rojas, and M. H. G. Tytgat, J. High Energy Phys. 03 (2018) 197.

[37] M. C. Gonzalez-Garcia, M. Maltoni, Y. F. Perez-Gonzalez, and R. Zukanovich Funchal, J. High Energy Phys. 07 (2018) 019.

[38] C. Bhm, D. G. Cerdeo, P. A. N. Machado, A. Olivares-Del Campo, and E. Reid, J. Cosmol. Astropart. Phys. 01 (2019) 043. 


\section{University Library}

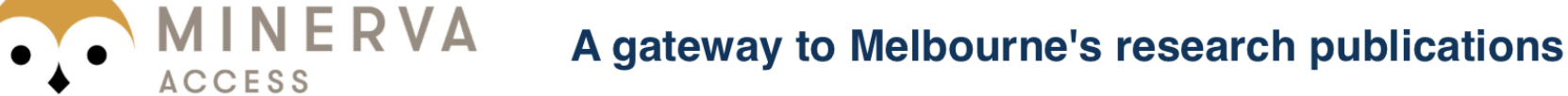

Minerva Access is the Institutional Repository of The University of Melbourne

Author/s:

Newstead, JL;Lang, RF;Strigari, LE

Title:

Atmospheric neutrinos in next-generation xenon and argon dark matter experiments

Date:

2021-12-22

Citation:

Newstead, J. L., Lang, R. F. \& Strigari, L. E. (2021). Atmospheric neutrinos in nextgeneration xenon and argon dark matter experiments. PHYSICAL REVIEW D, 104 (11), https://doi.org/10.1103/PhysRevD.104.115022.

Persistent Link:

http://hdl.handle.net/11343/297495 